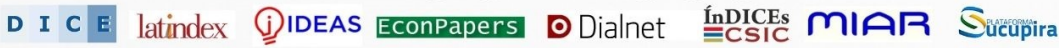

\title{
DIVERSIDAD FAMILIAR EN EL CONTEXTO ESCOLAR
}

Ana Alemán Aledo

Universidad de Murcia, España

https://orcid.org/0000-0001-5430-7372

Correspondencia: ana.a.a@um.es

Para citar este artículo puede utilizar el siguiente formato:

Ana Alemán Aledo (2021): "Diversidad familiar en el contexto escolar", Revista DELOS, (Especial noviembre 2021). En línea:

https://doi.org/10.51896/DELOS/HWCX2927

\section{RESUMEN}

El presente trabajo tiene como objetivo primordial transmitir a docentes y familias la necesidad de trabajar la diversidad familiar desde los primeros años de vida, ofreciendo una serie de recursos y estrategias que favorecen la práctica docente y la cooperación familia- escuela. Para alcanzar este objetivo es necesario que ambos contextos colaboren estrechamente con el fin de conseguir que todos los tipos de familia sean valorados y respetados por todas las personas independientemente de la identidad de su familia propia. Se expondrá una breve revisión histórica de cómo han ido cambiando las familias a lo largo de los años, dando lugar a una gran diversidad de familias actuales. Concluyendo con un listado de intervenciones educativas y herramientas que permitan trabajar a nivel de aula y de centro la diversidad familiar.

Palabras clave: familia, escuela, cooperación, diversidad familiar, inclusión.

\section{ABSTRACT}

\section{FAMILY DIVERSITY IN THE SCHOOL CONTEXT}

The main objective of this work is to convey to teachers and families the need to work on family diversity from the first years of life, offering a series of resources and strategies that improve teaching and family-school cooperation. To achieve this objective, it is necessary for both contexts to collaborate closely in order to ensure that all types of family are valued and respected by all people regardless of the identity of their own family. There will be a brief historical review of how families have changed over the years, giving rise to a great diversity of current families. Concluding with a list of educational interventions and tools that allow family diversity to work at the classroom and center level.

Keywords: family, school, cooperation, family diversity, inclusion.

\section{INTRODUCCIÓN}

Actualmente existen dos contextos inmediatos en la educación de cualquier sujeto, el contexto familiar y el contexto escolar. Comprender en qué consiste educar a una persona podría 
suponer un alto grado de dificultad debido a las respuestas tan variadas y complejas que podrían surgir, pero en la mayoría, se podría encontrar que educar a una persona supone preparar al sujeto para vivir en sociedad a través de una interacción continua y simultanea entre el educador y el educado (Ruíz, 2010). Educar consiste en formar personas que alcancen el pleno desarrollo de todas sus capacidades, adquiriendo todos aquellos valores que les permita estar comprometidas con la sociedad en la que viven (Parada, 2010).

Siendo conscientes de que la infancia supone la etapa más importante en la vida del niño, ya que su desarrollo posterior va a depender de las experiencias y los aprendizajes que el niño obtenga de los 0 a los 6 años, se debe potenciar una cooperación positiva entre la familia y la escuela desde estas primeras edades. Para asegurar una cooperación válida y eficiente se debe hacer desde el respeto y el apoyo a la gran diversidad familiar actual, proporcionándole a todo tipo de familia el valor que se merece. Además, es necesario trabajar de forma explícita e implícita todos los tipos de familia en el contexto escolar, haciendo especial mención a aquellos tipos de familia presentes en las aulas del propio centro, transmitiendo así a todo el alumnado que ellos y sus familias son importantes para los demás.

De acuerdo con la idea fundamental de la importancia que tiene la cooperación familia- escuela, la educación de cualquier sujeto debe ser una tarea compartida entre la familia y la escuela en busca de un objetivo común centrado en el desarrollo integral del niño/a (Domínguez, 2010).

La educación supone un proceso demasiado complejo que no se debe dejar como responsabilidad única de los docentes, ya que, el niño convive desde el momento de su nacimiento en el seno familiar, vive con su familia las primeras experiencias, lo que supondrá para él multitud de aprendizajes. Posteriormente, cuando el niño comienza la escolaridad, pasará muchas horas en el contexto escolar con sus maestros y compañeros, lo que supondrá un aprendizaje continuo que abarcará contenidos conceptuales, procedimentales y actitudinales, promoviendo así, su desarrollo integral. No obstante, la familia continuará siendo el contexto educativo más inmediato del niño y en el que más tiempo pasará, es por ello que se habla de una necesidad inminente de que exista cooperación directa y continua entre familia y escuela, a través de una relación positiva, con intercambios de conocimiento regulares y la capacidad de eliminar diferencias para promover el progreso adecuado de un comportamiento en ambos contextos favorecedor del desarrollo del niño (Mérida, 2002). La comunidad educativa no solo considera que la cooperación familia y escuela es una necesidad básica para el adecuado desarrollo del niño, sino que también manifiesta el derecho que tiene la familia a participar de forma activa en la vida del centro educativo (Carmona, Parra y Gomariz, 2021). El contexto escolar debe ofrecer a las familias la posibilidad de participar en la vida del centro, ya que, el interés y la implicación de los progenitores incrementa notablemente la confianza del niño en la comunidad educativa y aumenta su autoestima (Belmonte, Bernardez y Conzi, 2020).

Así pues, para favorecer el desarrollo integral del niño es fundamental que ambos contextos cooperen estrechamente y de forma positiva, estableciendo cauces y mecanismos de relación y participación de las familias en el proceso educativo de su hijo, prestando especial atención a la diferenciación de roles entre docentes y padres/ madres (Domínguez, 2010). 
Centrando el tema pretendido, la familia supone un grupo de personas con una organización abierta y cambiante, unidos por un vínculo afectivo especial que los diferencia de los demás, siendo este biológico o no. Está compuesta por todos sus miembros como un único conjunto e incluye diferentes generaciones. Los miembros de la familia desempeñan roles diferentes, y estos además son cambiantes en función de cada situación, ejerciendo más o menos control o afectividad en cada caso. Es destacable que la familia es el primer contexto social, cuya función principal es la de educar y cubrir las necesidades básicas de alimentación, higiene, descanso, vestido, afectividad, protección... La base para el buen funcionamiento familiar es el apoyo, afecto y respeto entre sus miembros, independientemente de que convivan o no juntos, y continuamente estarán persiguiendo un mismo objetivo: mantenerse unidos y en equilibrio (Espinoza, 2020).

Realizando una breve revisión histórica sobre los grandes cambios que ha sufrido la familia con el transcurso de los años, es destacable que la familia ha experimentado multitud de modificaciones desde sus orígenes hasta nuestros días, pero actualmente sigue siendo el primer contexto educativo y social. Además, para los miembros de la sociedad, la familia supone un punto de partida y de retorno en cualquier momento de la vida, constituyendo un núcleo afectivo único e inigualable. De acuerdo con lo manifestado, la familia postmoderna que se conoce hoy en día, poco tiene que ver con la familia tradicional. Es por ello que se van a exponer los rasgos más esenciales de las familias a lo largo de la historia, que van a permitir concebir las modificaciones surgidas en las relaciones existentes dentro de los subsistemas familiares, especialmente en el subsistema conyugal y subsistema parental.

Haciendo una breve revisión histórica, en la familia tradicional, se encontraban relaciones parentales extensas, residencia unifamiliar y una estructura estable, siendo un régimen autoritario con sumisión de todos los demás miembros. Estas familias tenían un elevado número de hijos a los que consideraban como mano de obra que se debía encargar del patrimonio familiar a través del cuidado de las tierras, el ganado...; o para contraer matrimonio con los descendientes de otra familia que tuvieran bienes distintos a los suyos. La familia tradicional otorgaba un gran valor a la religión y era considerada una unidad de producción (Valdivia, 2008).

Entre los años 70-80 surge la familia moderna, la cual fue evolucionando con el paso del contexto rural al urbano dejando de ser unidad de producción para ser una unidad de consumo. Así pues, fue aumentando la movilidad física de las familias provocando de esta manera la reducción del número de parientes e hijos, dando paso a un tipo de familia más nuclear. La mujer también se incorporó al trabajo, existía una visión de consumismo y hedonismo dentro de la familia, y había menos estabilidad estructural (Valdivia, 2008).

Con el transcurso de los años, la familia ha experimentado numerosos cambios que han permitido la aparición de la familia postmoderna. Estas transformaciones de la familia se han visto reflejadas en la sociedad, y al mismo tiempo, la sociedad ha experimentado cambios reflejados en la familia, por lo que se podría decir que ambas se van desarrollando de forma paralela, afectando una a la otra. Se podría destacar que en el año 2005 se aprobaron dos leyes muy importantes en España, la Ley 15/2005, de 8 de julio, por la que se modifican el Código Civil y la Ley de Enjuiciamiento Civil en materia de separación y divorcio que modifica la Ley 30/1981, de 7 de julio, 
por la que se modifica la regulación del matrimonio en el Código Civil y se determina el procedimiento a seguir en las causas de nulidad, separación y divorcio; y la Ley 13/2005, de 1 de julio, por la que se modificó el Código Civil en materia de derecho a contraer matrimonio. Ambas leyes han tenido una gran influencia en las diversas tipologías de familia que han ido surgiendo en la última década (familia nuclear, familia monoparental, familia homoparental, familia reconstituida, familia adoptiva y familia sin hijos). Además, existe una gran caída de la nupcialidad y de la natalidad (por debajo del reemplazo generacional), aumento de abortos, aceptación de la ingeniería genética y aumento de la inestabilidad en la estructura familiar presentándose mayor número de divorcios y separaciones, todos estos cambios se han visto influidos por el aumento de autonomía y libertad de la mujer (Instituto de Política Familiar, 2019).

Pese a la revisión histórica descrita referente a las modificaciones que ha ido experimentando la familia a lo largo de los años, siempre ha existido diversidad familiar pero debido a la época los tipos de familia que se alejaban de cumplir las características de una familia tradicional se ocultaban o invisibilizaban, manteniendo ante las demás personas la apariencia de una familia común a su época (López et al., 2008).

\section{METODOLOGÍA}

A través de este trabajo se pretende dar respuesta a la necesidad urgente de incorporar de forma habitual la diversidad familiar y la coeducación en el aula, presentando una serie de estrategias y recursos que ofrezcan un apoyo necesario a los docentes, permitiéndoles dar respuestas a las necesidades actuales presentes en los centros educativos con la aplicación de una intervención didáctica y moral, continua y formativa a lo largo de toda la escolaridad. Esta intervención pretende mejorar los mecanismos de detección e intervención de desigualdades en relación a la diversidad familiar tan presente en la realidad actual de las aulas.

Tanto en el contexto escolar como en el familiar se debe apelar por la individual-formación social, para ello, el educador debe ser consciente de que no existen dos personas iguales, ni si quiera dos miembros iguales en la misma familia, cada persona tiene unas características, necesidades e intereses diferentes, por ello, el educador debe realizar una adaptación constante a las peculiaridades individuales de cada niño/a (Parada, 2010). Esta adaptación debe cubrir la necesidad y derecho de todo niño a trabajar dentro del contexto educativo el tipo de familia al que pertenece.

De acuerdo con las familias actuales, la gran relevancia del contexto familiar desde los primeros años de vida y la importancia de impulsar todos los aspectos incluidos en la Agenda 2030 aprobada en el año 2015 por la Organización de las Naciones Unidas (ONU), en la que se incluyen los Objetivos de Desarrollo Sostenible (ODS) suponiendo una oportunidad para que los países y sus ciudadanos emprendan un nuevo camino para mejorar la vida de todas las personas, en los centros educativos, se vuelve fundamental la idea de trabajar la diversidad familiar como una realidad inmediata en las aulas. Trabajar desde la diversidad familiar permitirá que los docentes y discentes conozcan y sean conscientes de la necesidad de respetar a todas las personas y familias por igual, independientemente de sus características, esta actuación fomentará las posibilidades de terminar 
con la desigualdad y la discriminación entre las personas (Aguado, 2010). Además, trabajar la diversidad familiar está estrechamente relacionado con la Educación en valores cívicos para la convivencia y la coeducación. No obstante, es un derecho de cualquier ser humano trabajar en la escuela el valor por el tipo de familia a la que pertenece, fortaleciendo su identidad y autoestima; del mismo modo, es un derecho del alumnado conocer la gran diversidad familiar existente entre sus compañeros/as, fomentando la tolerancia, el respeto y la equidad.

De acuerdo con la Agenda 2030, se debe destacar su objetivo número cuatro referente a una educación de calidad a través del cual se pretende alcanzar una educación inclusiva y equitativa de calidad para todos, promoviendo a través de ella diversas oportunidades de aprendizaje permanente. Considerando la educación como un derecho humano a través de la cual se permitirá alcanzar los conocimientos teóricos y prácticos necesarios para promover el desarrollo sostenible y los estilos de vida saludables, el conocimiento y el respeto a los derechos humanos incluyendo la igualdad de género, incrementando una cultura de paz y de no violencia que permitirá la mejora de la ciudadanía mundial y la valoración positiva de la diversidad cultural (Naciones Unidas, 2015).

Es indiscutible la necesidad de trabajar la diversidad familiar en las aulas desde las primeras edades para alcanzar una educación inclusiva y equitativa de calidad, pero para ello es necesario que los centros educativos propongan actuaciones docentes que promuevan la participación de las familias en la vida del centro. Es primordial que la cooperación entre familia y escuela comience por un conocimiento mutuo inicial que debe perdurar en el tiempo debido a que ambos constituyen dos contextos dinámicos que van cambiando continuamente, así como la necesidad de que conozcan las estrategias de colaboración necesarias en cada momento a lo largo del proceso educativo de los discentes (Aguado, 2010).

Existen diferentes tipos de participación de las familias en el contexto escolar que a menudo se puede ver limitada por la jornada laboral de los progenitores e incluso por su nivel educativo (Anabalón et al., 2008). Además, algunos autores afirman que en las primeras etapas educativas es donde mayor participación familiar existe, pues sus hijos/as demandan más la figura de sus progenitores, en cambio esta necesidad por parte de los discentes va disminuyendo progresivamente conforme van avanzando de nivel, y con ella, la participación de sus familias (Rivera y Milicic, 2006). De acuerdo con el estudio realizado por Sánchez, Reyes y Villaroel (2016), se podría decir que hay familias que aportan un apoyo educativo a sus hijos desde el hogar y además participan de forma activa en la vida del centro educativo, otras familias que participan en la vida del centro, y otras que tan solo presentan una participación limitada a la recepción de información sobre el proceso educativo de sus hijos/as. Es muy importante que el docente conozca los tipos de familia que tiene en su aula y cómo motivar su participación para conseguir que sean conscientes y exigentes de las posibilidades educativas del sistema educativo actual, y que sus expectativas coincidan con la finalidad real de las escuelas, buscando en ellas que participen de forma activa en la vida escolar y, además, que proporcionen el apoyo e incrementen la motivación en el niño hacia la escuela. Existe una estrecha relación entre la participación y las expectativas de los padres y madres sobre la educación de sus hijos. Del mismo modo, se podría afirmar que la participación de los progenitores y 
sus expectativas hacia el contexto escolar influyen notablemente en el rendimiento académico y en las expectativas del propio niño hacia la escuela (Sánchez, Reyes y Villaroel, 2016).

Aunque no existe homogeneidad en la participación desarrollada por parte de las familias, se puede hablar de una serie de actuaciones que se desarrollan desde el colegio para fomentar esta cooperación tan necesaria. En los centros educativos se desarrollan actividades de participación directa en las que la familia coopera en actividades que forman parte del proceso de enseñanzaaprendizaje (de forma sistemática o esporádica); actividades de participación no presencial en las que la familia colabora con el equipo docente desde casa para favorecer la adquisición de determinados contenidos trabajados en ambos contextos; actividades formales, a través de ellas los padres pueden participar en la Asociación de Madres y Padres de Alumnos (AMPA) o en el consejo escolar; actividades de formación que suponen la organización de actividades por parte del centro educativo para familias con el fin de trabajar algún tema específico que surja en el contexto escolar como una necesidad o temas de interés para las familias (charlas, talleres...); y actividades de información que pueden ser entendidas como la posibilidad de transmitir información por parte de los docentes a las familias a través de circulares, encuentros diarios en las entradas y salidas del aula, reuniones, entrevistas... Además, actualmente se ha vuelto fundamental el contacto entre el centro educativo y las familias a través de las nuevas tecnologías (adquisición de información a través de la página web del colegio, reuniones o entrevistas a través de plataformas como "Meet", "Skype", "Zoom"..., y el envío de circulares a las familias a través del correo electrónico o de las aplicaciones de mensajería instantánea). Otro tipo de actividades son las de formación que suponen la organización de actividades por parte del centro educativo para familias con el fin de trabajar algún tema específico que surja en el contexto escolar como una necesidad o temas de interés para las familias (charlas, talleres...). Las familias del alumnado pueden ser partícipes de diferentes tipos de actividades a lo largo del curso escolar.

\section{Implicaciones educativas en el contexto escolar para favorecer la diversidad familiar}

Siendo conscientes de la necesidad de que exista una cooperación activa, continua y favorable entre escuela y familia desde el comienzo de la escolaridad, y conociendo la gran variedad de familias existentes en la actualidad, es indiscutible la necesidad de incorporar a las familias a las actividades diarias desarrolladas en el centro desde su más pura heterogeneidad. Esta actuación por parte de las familias en las aulas y en el centro escolar de sus hijos permitirá que todos los miembros de la comunidad educativa conozcan las características, necesidades e intereses de cada una de ellas, procurando crear un vínculo de afectividad y apoyo común.

Por ello, es necesario plantear estrategias basadas en los principios de Coeducación entendida como la educación impartida a las personas al margen de todos los roles y estereotipos impuestos por la sociedad; eliminación de todo tipo de violencia, actuando desde la paz y el respeto; y transmisión de valores alejados de la idea de que la familia tradicional es la única válida (Federación de enseñanza de CC. OO de Andalucía, 2010).

Una intervención educativa basada en estos principios aporta numerosos beneficios al desarrollo integral del niño: adquisición de autonomía y capacidad crítica, desarrollo de la afectividad 
y la creatividad, potenciación expresiva y comunicativa, el respeto y cuidado a todos los seres vivos y al entorno, resolución de problemas de la vida cotidiana, y la normalización de la participación de hombres y mujeres en las tareas domésticas y no domésticas en igualdad. Es muy importante que los niños y niñas desde las primeras edades sientan la diversidad familiar como una realidad habitual (Aguado, 2010).

Para trabajar la diversidad familiar de forma eficiente se debe prestar especial atención al currículo oculto, pues en muchas ocasiones, los docentes de forma no consciente transmiten a su alumnado valores totalmente diferentes a los pretendidos, es por ello que a continuación se exponen una serie de aspectos a tener en cuenta en la vida del centro educativo:

Los docentes deben recibir una formación permanente que les permita alcanzar una conciencia plena sobre la diversidad familiar actual.

Desde los centros educativos se debe aprovechar la inquietud de las familias por conocer juguetes, cuentos, películas... adecuadas a la edad de sus hijos e hijas para ofrecerles recursos coeducativos que contribuyan a su desarrollo integral. Esto se hará a través de las escuelas de padres, las tutorías, talleres de formación para padres...

Se debe utilizar un lenguaje inclusivo en los textos formales del centro y en las comunicaciones enviadas a las familias.

- El docente debe cuidar el vocabulario utilizado con los niños y niñas evitando normalizar a las familias nucleares dejando en segundo lugar a los demás tipos de familia.

- Se deben potenciar los contenidos relacionados con la diversidad familiar de forma transversal y explícita.

Uso de metodologías innovadoras y de las nuevas tecnologías que potencien la motivación y el interés en el alumnado.

Creación y uso de materiales que permitan trabajar el tema desde perspectivas teóricas y lúdicas.

- Se deben revisar todos los recursos didácticos (cuentos, impresos, videos, juguetes...) para suprimir aquellos que no incluyan a los diversos tipos de familias.

Los docentes deben ser conocedores de la influencia de los medios de comunicación en los niños y niñas, sobre todo la televisión, ayudándolos a analizar los mensajes sexistas y los que no incluyen una diversidad familiar de forma crítica, promoviendo la igualdad en dibujos animados, películas, anuncios...

En el centro educativo se trabajará el 15 de mayo el Día Internacional de la Familia que fue establecido por las Naciones Unidas. Se debe de tener en cuenta que celebrar el día del padre y de la madre puede causar emociones negativas en niños/as que no cuentan con esta figura en su familia.

De forma más concreta, se van a exponer una serie de intervenciones educativas que fomentan la igualdad entre los tipos de familia a través de rutinas desarrolladas en el contexto escolar y que van a permitir trabajar de forma transversal los valores deseados para lograr una educación de calidad que se preocupa por la inclusión de todos los miembros de la sociedad. Algunas propuestas son: 
- $\quad$ En todas las aulas de Educación Infantil y Educación Primaria se podría incorporar un día del cuento a la semana, de forma quincenal o al mes, en el que se narre una historia adaptada a cada etapa educativa relacionada directamente con la diversidad familiar, pudiendo incorporar una actividad posterior o un debate. En edades más avanzadas como en la Educación Secundaria podrían ser los propios alumnos los que busquen historias reales o leyendas relacionadas con la diversidad familiar (cómo se ha tratado a lo largo de la historia y actualmente, análisis de carencias y/o potencialidades de cada tipo de familia, investigaciones sobre el porcentaje de familias en España de cada tipo...)

Se podrían desarrollas talleres o jornadas de convivencia en el aula con la colaboración de las familias a través de los cuales se trabajen contenidos relacionados con la diversidad familiar, las actividades planteadas dependerán de la edad del alumnado. En Educación Infantil y en Educación Primaria, los alumnos/as podrían preparar una caja con fotografías, objetos... sobre su familia y exponerla en el aula con la colaboración de algún familiar para ser conscientes de las diferencias que existen entre las familias incluso dentro del mismo tipo. En Educación Secundaria los alumnos con la ayuda de algún familiar si lo desean, podrían elaborar un árbol genealógico a mano alzada o incluso a través de las nuevas tecnologías.

La diversidad familiar se podría trabajar a través de una unidad didáctica que tenga como hilo conductor los tipos de familia o incluso un tipo de familia en particular y a partir de ese, trabajar el resto. También, se podría desarrollar un proyecto de aula que se inicie desde el concepto "Familia" y sea el propio alumnado el que investigue sobre los tipos de familia, sus características, porcentajes, potencialidades y carencias, famosos con tipos de familia diferentes a la familia tradicional...

Los grupos interactivos podrían suponer una metodología muy válida para trabajar la diversidad familiar. Se podrían realizar investigaciones sobre las familias dentro del aula con el alumnado dividido en grupos de trabajo y con un familiar u otra persona de la comunidad educativa por grupo para guiarlos si lo necesitan. Estas investigaciones dependerán de la edad, los más pequeños podrían buscar en revistas, periódicos u otros materiales impresos diferentes tipos de familia e ir creando un collage o mural; y el alumnado de edades más avanzadas podría realizar investigaciones de diferente índole con datos reales proporcionados por el Instituto de Política Familiar o el Instituto Nacional de Estadística.

Existen numerosos recursos que permiten trabajar la diversidad escolar en el aula de Educación Infantil, Educación Primaria y Educación Secundaria. A continuación, a modo de ejemplo se expondrán algunos de ellos:

Literatura sobre diversidad familiar. Se podrían destacar cuentos como: Cada familia a su aire, Mi familia es de otro mundo, Un puñado de botones, Paula tiene dos mamás, Familiario.

Aplicaciones para crear juegos y videos sobre la diversidad familiar: Genially, Canva, Educaplay, Powton. 
- Aplicaciones para recoger información sobre los conocimientos del alumnado a través de cuestionarios: Quizizz, Blooket, Kahoot.

Aplicaciones para que el alumnado pueda crear videos y presentaciones relacionados con el tema: Tik- Tok, Vivavideo, Inshot, Kinemaster, Google Slides, Prezi.

Aplicaciones para crear árboles genealógicos: Family free builder, Family Search,Ancestral Quest Basis. O incluso en edades avanzadas (Bachillerato, Formación Profesional o incluso universidad) podrían crear un genograma de su familia que les permitirá observar patrones hereditarios y factores psicológicos a través de programas como Genopro.

Videos y documentales. En Youtube aparece una gran variedad de documentales en los que aparecen familias reales muy diversas, por ejemplo: That's Family, familiar- mente (diversidad familiar).

Videos y canciones. En Youtube aparecen una gran cantidad de videos y canciones para los más pequeños, por ejemplo: El baile de las familias, Todas las familias son diferentes.

\section{CONCLUSIONES}

Como principales conclusiones de todo lo expuesto se hace alusión a que la familia y la escuela suponen los dos primeros contextos educativos y sociales de cualquier niño/a, por ello ambos, deben cooperar estrechamente durante toda la escolarización de los discentes. Esta cooperación debe ser desarrollada de forma continua y positiva, desde el respeto y el apoyo mutuo, compensando las deficiencias que puedan surgir en cualquiera de los dos contextos.

El centro educativo debe procurar que las familias se respeten entre sí, independientemente de la tipología familiar que presenten. Ya que, los valores de respeto y apoyo hacia los demás son valores básicos de convivencia que deben ser transmitidos a los niños/as. Para que esto ocurra, las familias deben conocerse entre sí y participar en las actividades propuestas por el centro con el fin de que todas las familias convivan y colaboren juntas con el objetivo de promover el desarrollo global del niño/a. Como idea final, se debe destacar que existen multitud de recursos y estrategias que ayudarán al docente a mejorar la inclusión desde las aulas, pero no puede olvidar que los medios utilizados para disminuir las situaciones de desigualdad no pueden ser medios que conduzcan al incremento de esta problemática, ya que, en ocasiones se les exige a las familias participar a través de intervenciones que se encuentran muy alejadas de su realidad.

\section{REFERENCIAS}

Aguado, L. (2010). Escuela inclusiva y diversidad de modelos familiares. Revista Iberoamericana de Educación, 53 (6), 1-11. https://rieoei.org/historico/deloslectores/3377Aguado.pdf

Anabalón, Mirian., Carrasco, S., Díaz., D., Gallardo, C., y Cárcamo, H. (2008).

El compromiso familiar frente al desempeño escolar de niños y niñas de educación general básica en la ciudad de chillán. Revista Horizontes Educacionales, 13 (1), 11-21. https://www.redalyc.org/pdf/979/97912446001.pdf 
Belmonte, M.L., Bernardez, A., y Conzi, Q.T. (2020). La relación familia-escuela como escenario de colaboración en la comunidad educativa. Revista Valore, 5. https://www.researchgate.net/publication/343126779 LA RELACION FAMILIAESCUELA COMO ESCENARIO DE COLABORACION EN LA COMUNIDAD EDUCATIV $\underline{A}$

Carmona Sáez, P., Parra Martínez, J. y Gomariz Vicente, V. (2021). Participación de las familias de alumnado con apoyos y atenciones diferentes: un estudio en un contexto multicultural. Revista de Investigación Educativa, 39(1), 49-69. http://dx.doi.org/10.6018/rie.386551

Domínguez, S. (2010). La Educación, cosa de dos: La escuela y la familia. Revista digital para profesionales de la enseñanza, 8, 1-15. https://www.feandalucia.ccoo.es/docu/p5sd7214.pdf

Espinoza, A. (2020). Estilos de socialización parental en una muestra de adolescentes chilenos. Revista de Psicología Clínica con Niños y Adolescentes, 7 (1), 46- 51. https://www.revistapcna.com/sites/default/files/1748-2.pdf

Federación de enseñanza de CC. OO de Andalucía (2010). Coeducación en Educación Infantil. Revista digital para profesionales de la enseñanza, 9. https://www.feandalucia.ccoo.es/docu/p5sd7366.pdf

Instituto de Política Familiar (2019). Informe Evolución de la Familia en España 2019. http://www.ipfe.org/Espa\%C3\%B1a/Documentos/IPF

Ley 15/2005, de 8 de julio, por la que se modifican el Código Civil y la Ley de Enjuiciamiento Civil en materia de separación y divorcio. Boletín Oficial del Estado, 163, de 9 de julio de 2005, 24458 a 24461. https://www.boe.es/eli/es///2005/07/08/15

Ley 13/2005 del 1 de julio, por la que se modificó el Código Civil en materia de derecho a contraer matrimonio. Boletín Oficial del Estado, 157, de 2 de julio de 2005, 23632 a 23634. https://www.boe.es/buscar/doc.php?id=BOE-A-2005-11364

Ley 30/1981, de 7 de julio, por la que se modifica la regulación del matrimonio en el Código Civil y se determina el procedimiento a seguir en las causas de nulidad, separación y divorcio. Boletín Oficial del Estado, 172, de 20 de julio de 1981, 16457 a 16462.

https://www.boe.es/eli/es///1981/07/07/30

López, F., Díez, M., Morgado, B., y González, M.M. (2008). Educación infantil

y diversidad familiar. XXI, Revista de Educación, 10, 111-122. http://rabida.uhu.es/dspace/bitstream/handle/10272/2145/b15480057.pdf?sequence $=1$

Mérida, R. (2002). Un espacio de encuentro entre la escuela y las familias: la

escuela de madres y padres. Revista de Ciencias de la Educación, 192, 1-27. https://redined.mecd.gob.es/xmlui/bitstream/handle/11162/32528/2002 CienciasEducaci\%c3 \%b3n 19231923 M\%c3\%a9rida\%20Serrano.pdf?sequence=1\&isAllowed=y

Naciones Unidas (2015). Objetivos de desarrollo sostenible. La asamblea general adopta la Agenda 2030 para el Desarrollo https://www.un.org/sustainabledevelopment/es/2015/09/la-asamblea-general-adopta-laagenda-2030-para-el-desarrollo-sostenible/ 
Parada, J.L. (2010). La educación familiar en la familia del pasado, presente y futuro. Educatio Siglo XXI, 28 (1), 17-40. https://revistas.um.es/educatio/article/view/109711/104401

Rivera, M., y Milicic, N. (2006). Alianza familia-escuela: percepciones, creencias, expectativas y aspiraciones de padres y profesores de enseñanza general básica. Psykhe, 15 (1), 119-135. https://www.redalyc.org/pdf/967/96715110.pdf

Ruíz, P.M. (2010). El rol de la familia en la educación. Revista digital para profesionales de la enseñanza, 10, 1-12. https://www.feandalucia.ccoo.es/docu/p5sd7489.pdf

Sánchez, A., Reyes, F., y Villaroel, V. (2016). Participación y expectativas de los padres sobre la educación de sus hijos en una escuela pública. Estudios Pedagógicos XLII, 3, 347-367. https://scielo.conicyt.cl/pdf/estped/v42n3/art19.pdf

Valdivia, C. (2008). La familia: concepto, cambios y nuevos modelos. La Revue du Redif, 1, 15-22. https://mimateriaenlinea.unid.edu.mx/dts cursos $\mathrm{mdl} / \mathrm{lic} / \mathrm{DE} / \mathrm{PF} / \mathrm{AM} / 05 /$ cambio s.pdf 